\title{
Réalisation d'une station automatique d'enregistrement des facteurs physico-chimiques dans la zone des marées
}

\author{
Francis BENARD \\ Laboratoire Maritime, Faculté des Sciences de Caen, Caen, France
}

\begin{abstract}
Realization of a station for automatical registration of physico-chemical factors in the intertidal zone. The automatic station is a device for the investigation of ecological parameters in the intertidal zone $(10 \mathrm{~m})$. The paper describes the electronic system design and the techniques used to protect the components from the ambient stresses. Parameters studied are: temperature, pressure, velocity and direction of currents, energy of light, chlorinity, $\mathrm{pH}$, redox-potential, oxygen content and turbidity. The installation technique and problems encountered are discussed.
\end{abstract}

\section{INTRODUCTION}

Pour acquérir une meilleure connaissance des conditions écologiques précises règnant dans la zone interditale, l'auteur présente la première réalisation d'une station immergée autonome enregistrant automatiquement une série de grandeurs physiques ou chimiques règnant dans le milieu marin.

Afin d'étayer systématiquement les causes de la répartition des organismes végétaux et animaux sur les littoraux à marées, nous avons cherché à élaborer des appareillages et méthodes d'étude in situ d'une série de paramètres physico-chimiques représentatifs. Dans un travail ultérieur (BÉNARD en imp.), nous proposons une étude systématique des relations entre les grandeurs physiques ou chimiques et les incidences biologiques réelles. Concrètement, nous y décrivons une station portative simple permettant le relevé d'un certain nombre de grandeurs par lecture directe sur appareils de mesure.

La lourde servitude de la surveillance permanente, l'impossibilité pratique de suivre les phénomènes à marée haute, donc d'établir la réalité d'un cycle continu de marée, nous a logiquement menés à envisager une méthode d'enregistrement.

Destinée essentiellement aux études biologiques une telle installation doit être la plus simple possible pour des raisons de prix, d'entretien, de manipulation et de réglage. Nous nous sommes efforcés de toujours choisir une solution simple et «fiable» au détriment de procédés peut-être plus raffinés ou précis mais plus délicats aussi. 


\section{CHOIX D'UNE IMPLANTATION DES ÉLËMENTS}

Différentes conceptions générales sont possibles (Fig. 1):

(a) Séparation d'un ensemble "sondes» placé in situ et d'un ensemble «récepteursenregistreur» sur le rivage (1); la liaison étant assuré par un câble. La nécessité d'amplificateurs intermédiaires pour certains signaux faibles conduit à une dispersion

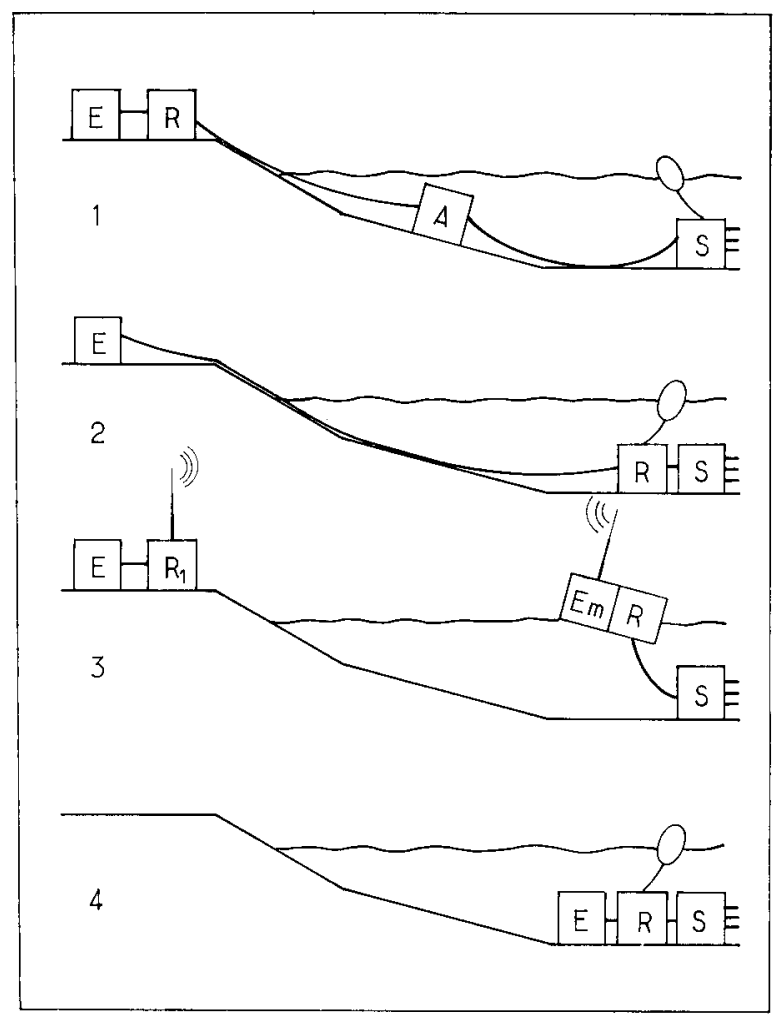

Fig. 1: Implantations générales; S: ensemble porte sondes; R: Récepteurs; E: Enregistreurs; A: amplificateur intermédiaire; Em: émetteur hertzien; $\mathrm{R}_{1}$ : récepteur hertzien

trop grande des différents éléments. D’autre part, le câble limite obligatoirement ie rayon d'action d'une telle installation. Une variante serait de séparer un ensemble «sonde-récepteurs» et un ensemble «enregistreur» (2); les servitudes resteraient les mêmes.

(b) Séparation d'un ensemble «sondes-récepteurs» placé in situ (3) les signaux étant repris par un émetteur hertzien avec antenne bouée; l'ensemble «récepteursenregistreurs» est au rivage ou au Laboratoire. Cette solution, la plus élégante, est délicate de réalisation et surtout très onéreuse. Elle demande également une assistance technique dont disposent rarement les laboratoires maritimes. Il serait enfin difficile de réaliser un émetteur suffisamment robuste pour résister aux chocs au moment des 
immersions et des émersions. Un émetteur immergé et l'antenne seule en surface empêcheraient l'accord correct du câble de transmission.

(c) Réunion des sondes, récepteurs et enregistreurs en un seul ensemble placé in situ. Bien qu'apparemment très vulnérable une telle installation réduit au minimum les complexités électroniques et ne pose en réalité que des problèmes mécaniques et particulièrement d'étanchéité. C'est cette solution qui a été choisie.
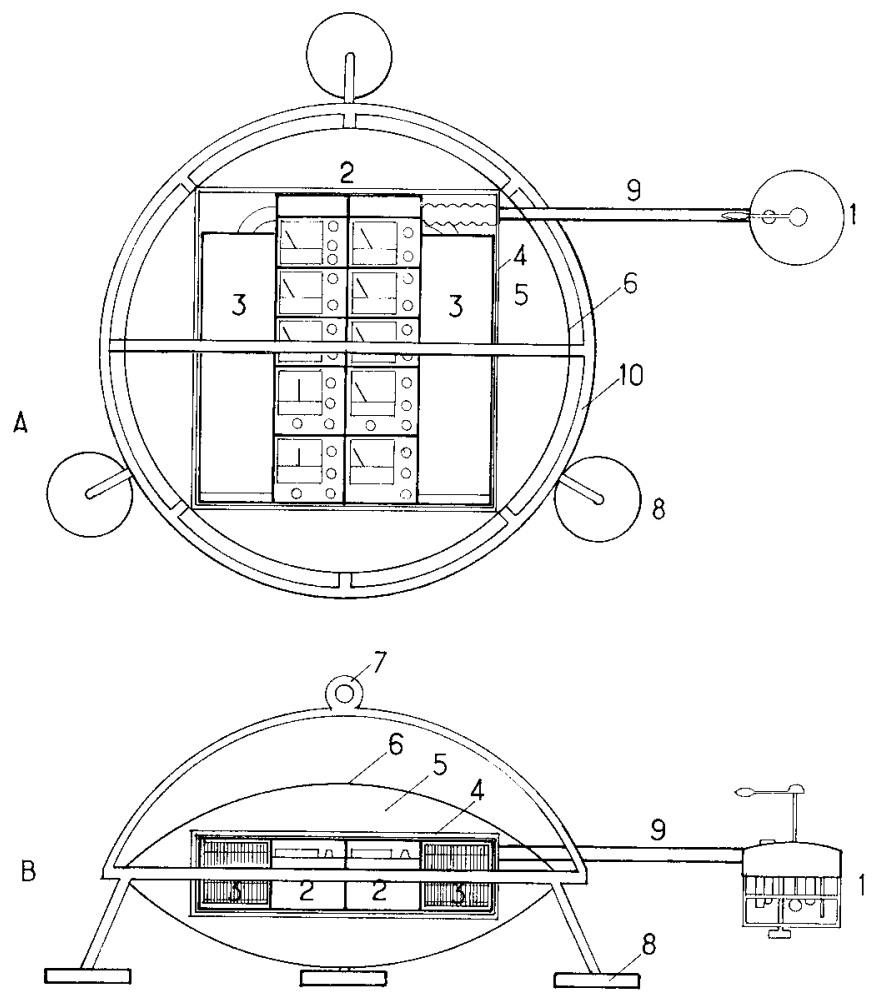

Fig. 2: Vue d'ensemble; A: vue supérieure; B: vue latérale; (1) porte sondes; (2) récepteurs; (3) enregistreurs; (4) carter; (5) ballast; (6) coupoles; (7) armature de manutention; (8) pieds lestés; (9) bras porte sondes

\section{DESCRIPTION GÉNÉRALE}

(a) Protection. Les différents composants sont regroupés dans un carter étanche (4) assurant la protection mécanique et l'étanchéité (Plexiglas ou Altuglas de $1 \mathrm{~cm}$ (Fig. 2).

Deux coupoles opposées (6) forment une protection mécanique directe externe sans marche d'étanchéité. Elles sont en Altuglas de $4 \mathrm{~mm}$. L'espace entre les coupoles et le carter (5) est utilisé comme waterballast par entrée d'eau au cours de l'immersion.

(b) Manipulation et stabilis ation. Le transport et le largage se font 
grâce à une armature tubulaire circulaire métallique (7) portant 3 pieds terminés par des masses de plomb (8) formant un lest bas. A l'anneau supérieur s'amarre un câble de manoeuvre en nylon. En service une bouée de surface signale le lieu de station.

(c) Ensemble porte-sondes (1). Nous avons recherché un compromis entre un rassemblement aussi serré que possible des sondes et une dispersion suffisante pour éviter les interférences hydro-dynamiques. Il est constitué d'un boîtier cylindrique de diamètre $20 \mathrm{~cm}$ et hauteur $6 \mathrm{~cm}$ en Altuglas de $1 \mathrm{~cm}$. A la face supérieure nous avons

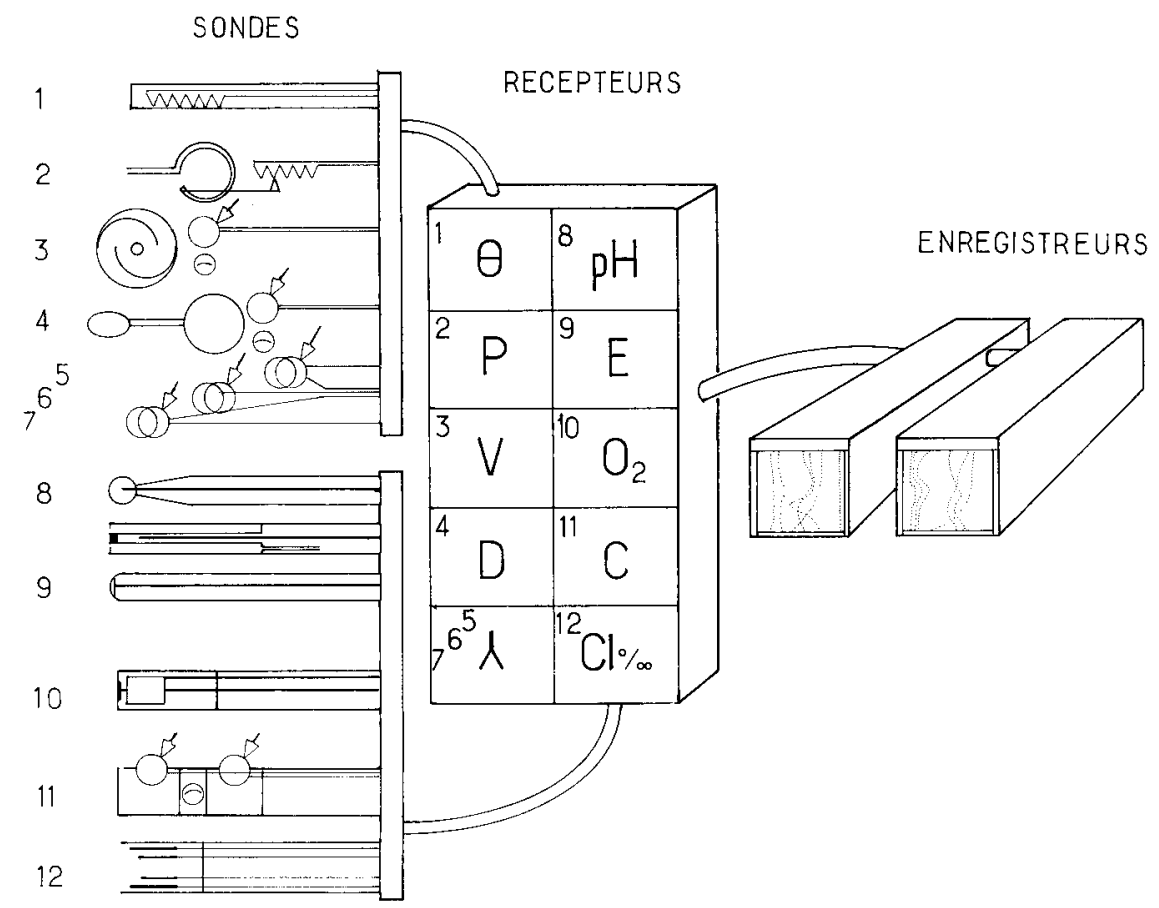

Fig. 3: Diagramme des unités. Les numéros de 1 à 12 correspondent aux sondes et aux récepteurs. (1) $\theta$ : température; (2) P: pression; (3) v: vitesse du courant; (4) D: direction du courant; (5-6-7) $\lambda$ : énergie lumineuse; (8) $\mathrm{pH}$; (9) $\mathrm{E}$ : potentiel redox; (10) $\mathrm{O}_{2}$ : pression d'Oxygène; (11) $\mathrm{C}$ : charge de turbidité; (12) $\mathrm{Cl} \%$ : chlorinité

placé la girouette indiquant la direction du courant et les trois cellules de mesure des radiations lumineuses. A la face inférieure sont placées les sondes de température, salinité, $\mathrm{pH}, \mathrm{E}$ redox, concentration d'O $\mathrm{O}_{2}$, turbulence, néphélométrie et pression. Deux arceaux circulaires assurent une protection de l'ensemble.

Le bras porte-sondes comprend un bras métallique articulé en 2 points permettant de disposer le porte-sondes dans un plan parallèle au sol de 0 à $70 \mathrm{~cm}$ de hauteur. Il livre passage au câble multifilaire vers les récepteurs. La protection est assurée par une gaine en polyvinyle souple.

(d) Récepteurs. Les récepteurs sont groupés en une double rangée de racks emboîtables, normalisés aux dimensions de $144 \mathrm{~mm} \times 144 \mathrm{~mm}$ et $144 \mathrm{~mm} \times 100 \mathrm{~mm}$ 
selon le volume des circuits électroniques correspondants. Cette station étant encore expérimentale chaque chaîne de mesure comprend un galvanomètre de contrôle permettant les règlages. En principe chaque récepteur possède son alimentation autonome (piles au mercure).

(e) Enregistreurs. L'unité d'enregistrement est constituée de deux potentiomètres enregistreurs multivoies $(2 \times 6$ voies) à pointés successifs. La largeur utile du diagramme est de $10 \mathrm{~cm}$, la précision de $0,5 \%$ permet théoriquement de distinguer 200 états. La vitesse de déroulement réglable a été fixée à $10 \mathrm{~cm} / \mathrm{h}$, le cycle de pointés est de 12 secondes pour l'ensembles des 12 canaux de mesure. L'alimentation des potentiomètres nécessite une source alternative pour la synchronisation des moteurs.

En principe la consommation des capteurs et récepteurs est faible et des piles incorporées suffisent pour de longues durées de fonctionnement ( 5 jours). Pour les potentiomètres enregistreurs nécessitant une tension alternative de 50 périodes nous utilisons: soit une liaison au rivage par un câble trifilaire branché sur le réseau de distribution, soit une batterie d'accumulateurs $(24 \mathrm{~V})$ séparée possédant un convertisseur continu-alternatif stabilisé pouvant fournir $40 \mathrm{~V}$. A. L'autonomie prévue est de cinq jours.

Dans l'avenir nous pensons incorporer à la station elle-même l'alimentation alternative par l'utilisation de batterie à haut rendement à l'Argent.

\section{GRANDEURS ET VOIES DE MESURE}

(a) Choix des grandeurs mesurées. A titre expérimental nous avons retenu dans cette première réalisation la mesure des grandeurs suivantes:

$\begin{array}{lllll}\text { Température } & 0 & & \text { Chlorinité } & \mathrm{Cl} \% 00 \\ \text { Pression } & \text { P. } & \text { Indice de Sorensen } & \mathrm{pH} \\ \text { Vitesse du courant } & \text { V. } & & \text { Potentiel redox } & \mathrm{E} \\ \text { Direction du courant } & \mathrm{D} . & & \text { Pression d'Oxygène } & \mathrm{O}_{2} \\ \text { Rayonnement sur } & 300-470 \mathrm{~nm} & \lambda_{\mathrm{B}} & \text { Charge de Turbidité } & \mathrm{C} \\ & 470-570 \mathrm{~nm} & \lambda_{\mathrm{V}} & & \\ & 570-800 \mathrm{~nm} & \lambda_{\mathrm{R}} & & \end{array}$

Ce choix représente naturellement un programme large où des suppressions ou des substitutions sont réalisables.

(b) Temperature $\theta$. Sonde: résistance de platine $100 \Omega$ à $0^{\circ} \mathrm{C}$ protégé par un tube métallique; gamme de $-10 \mathrm{a}+40^{\circ} \mathrm{C}$; incertitude: $0,2^{\circ} \mathrm{C}(\mathrm{p} .=0,9)$. La dérive est négligeable pour des périodes de plusieurs mois.

(c) Pression P. Sonde: capteur à résistance variable (potentiomètre microfriction) couplée à un tube de Bourdon. Une cartouche de verre poreux atténue les à-coups brusques de pression. Récepteur: mesure de la variation de tension; gamme de 0 à 1200 mbar (= 0 à $12 \mathrm{~m}$ d'eau); incertitude: \pm 10 mbar.

(d) Vitesse du courant. Sonde: moulinet de Savonius (diamètre $60 \mathrm{~mm}$ ). La mesure de la vitesse de rotation comprend un dispositif particulier; la face infé- 
rieure du moulinet comprend à la périphérie des secteurs alternativement blancs et noirs. Une ampoule à filament émet de la lumière réfléchie vers une photo-résistance à chaque passage d'un secteur blanc. Lampe et photo-résistance sont situées derrière une lame de verre; le rotor est entièrement libre en rotation.

Récepteur: totalisateur électronique indiquant instantanément la cadence d'émission des tops à partir de la sonde; gamme de mesure 0 à $1 \mathrm{~m} / \mathrm{s}$; incertitude $=1 \mathrm{~cm} / \mathrm{s}$ $(\mathrm{p}=0,9)$.

(e) Direction du courant. Sonde: girouette à penne constituée (1) d'un ellipsoïde de révolution (2) selon LACRoIx \& LAUBier (1962) pour éliminer les mouvements en lacets (Fig. 4). La girouette est solidaire d'un boîtier (3) contenant un disque

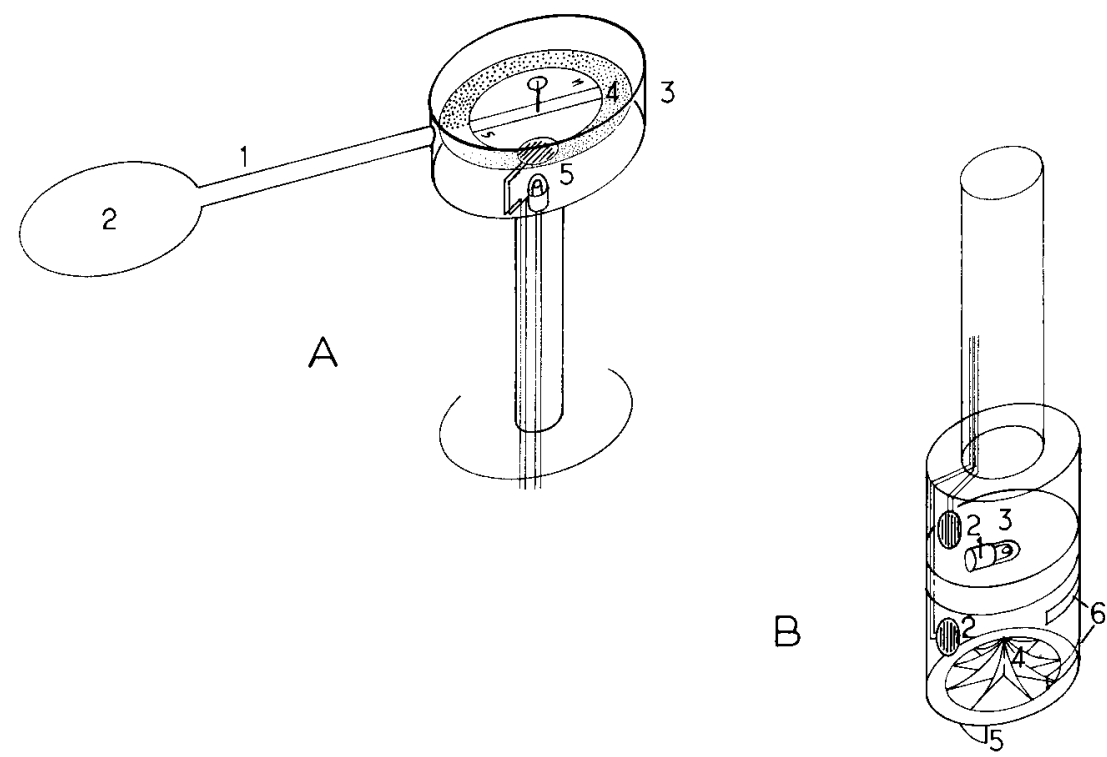

Fig. 4: A: girouette de direction; (1) bras de girouette; (2) ellipsoïde; (3) boïtier mobile; (4) coin de Golberg et barreau aimanté; (5) couple lampe-photorésistance. B: sonde néphélométrique; (1) lampe à incandescence; (2) photorésistances; (3) milieu de référence; (4) turbine centrifuge;

(5) moulinet de Savonius; (6) évents

(4) portant un coin de Goldberg circulaire à la périphérie et un barreau aimanté selon un diamètre. La densité optique du coin est mesurée par un couple lampe-photorésistance (5). Dans ces conditions la mesure revient à évaluer l'angle fait par la girouette (direction du courant) et le nord magnétique. Ce dispositif permet un largage aveugle à partir du bateau et une position quelconque de la station par rapport au nord. Récepteur: mesures de la variation de tension; gamme: 0 à $400 \mathrm{~g}$; incertitude: $\pm 2 \mathrm{~g}$ $(\mathrm{p}=0,9)$.

(f) Rayonnement. Sonde: un boîtier unique comprend 3 cellules photorésistantes portant chacune un filtre de verre. Le spectre visible est divisé en 3 régions: $300-470 \mathrm{~nm} ; 470-570 \mathrm{~nm} ; 570-800 \mathrm{~nm}$. Récepteur: mesure de la variation de tension. Nous avons choisi d'exprimer l'intensité des rayonnements en énergie (cal $\left./ \mathrm{m}^{2} / \mathrm{mn}\right)$. Un 
étalonnage préalable de chaque filtre au pyrhéliomètre a été effectué. Les résultats sont donc exprimés par la quantité d'énergie ayant traversé chaque filtre. Gamme: 0 à 10 $\mathrm{cal} / \mathrm{m}^{2} / \mathrm{mn}$; incertitude: $\pm 1 \mathrm{cal} / \mathrm{m}^{2} / \mathrm{mn}(\mathrm{p}=0,9)$.

(g) $\mathrm{Ch}$ lorin i té $\mathrm{Cl} \%$. Sonde: cellule de conductivité à 4 électrodes de platine en Altuglas (Volume de l'échantillon $2 \mathrm{~cm}^{3}$ ); alimentation de la sonde: signal alternatif sur 3000 hertz à intensité constante; récepteur: millivoltmètre à forte impédance d'entrée permettant de négliger les résistances parasites d'électrodes polluées; gamme: $\mathrm{C}-20 \mathrm{Cl} \%$; incertitude $\pm 0,10 \%(\mathrm{p}=0,9)$. Les résultats demandent une correction de température sur abaque.

(h) $\mathrm{pH}$. Sonde: électrode de verre à bulbe de type industriel. Sa résistance aux chocs est grande et sa résistance électrique faible $(50 \mathrm{M} \Omega)$. Electrode de référence au calomel autopressurisée jusqu'à 1000 mbar. Récepteur: voltmètre adaptateur d'impédance à forte résistance d'entrée $(1000 \mathrm{M} \Omega)$ à transistors à effet de champ; gamme: de 2 à 9 unités $\mathrm{pH}$; incertitude: $\pm 0,1$ unité $(\mathrm{p}=0,9)$. La dérive est de l'ordre de 0,2 unité pour une semaine d'utilisation constante. La correction de température se fait sur abaque.

(i) Potentiel redox. Sonde: électrode de platine de type industriel et électrode de référence commune avec celle de $\mathrm{pH}$; récepteur: Voltmètre adaptateur d'impédance à transistor à effet de champ; gamme: $-200 \mathrm{mv}$ à $+200 \mathrm{mv}$; incertitude: $\pm 2 \mathrm{mv}(\mathrm{p}=0,9)$. La valeur réelle du redox et $\mathrm{du} \mathrm{rH}$ est calculée sur un abaque.

(j) Concentration en oxygène. Sonde: électrode ampéromètrique (anode d'argent et cathode de platine). Courant ampéromètrique de $0,8 \mathrm{v}$ par un polariseur à pile au mercure; récepteur: voltmètre électronique à faible chute de tension; gamme: 0 à $100 \%$ de la saturation; incertitude $\pm 2 \%(\mathrm{p}=0,9)$; correction de température sur abaque. La masse d'oxygène par litre en fonction de $\mathrm{Cl} \%$ et $\theta$ peut-être calculée par un abaque.

(k) Charge (turbidité). Sonde: cellule mesurant la lumière réfractée par l'échantillon à partir d'une lampe (Fig. 4) (1). Un montage en double faisceau minimise les variations d'intensité de la source lumineuse. Les éléments photo sensibles sont des cellules photo résistantes. La solution de référence est une suspension gélifiée (3) d'un échantillon d'eau de mer locale contenant par analyse pondérale $1 \mathrm{~g} / \mathrm{l}$ de particules. La réponse de la cellule néphélométrique établie expérimentalement suit passablement la loi de LAMBERT. Quoiqu'il en soit, on sait combien cette méthode doit être utilisée prudemment. Pour l'instant, nous avons cherché un moyen d'indication permettant de suivre les tendances d'évolution de ce facteur en fonction des autres. Un dispositif rotatif (4) empruntant l'énergie au mouvement de la mer par un moulinet de SAvonius permet un renouvellement de l'échantillon par des évents (6).

Récepteur: montage différentiel fournissant le rapport entre les signaux issus des deux cellules; gamme de 0 à $5 \mathrm{~g} / \mathrm{l}$; incertitude: $\pm 0,4 \mathrm{~g} / 1(\mathrm{p}=0,9)$.

\section{RÉSULTATS PRÉLIMINAIRES ET PROBLÈMES COMPLÉMENTAIRES}

L'achèvement de la construction étant récent, nous avons surtout procédé à des essais de la sécurité mécanique. Le premier écueil est certainement le poids de l'en- 
semble: le carter extérieur ayant comme dimensions extérieures $62 \times 66 \times 18$ la poussée hydrostatique est de l'ordre de $70 \mathrm{~kg}$. Or, les potentiomètres enregistreurs, les circuits électroniques et le carter ne réalisent à eux seuls qu'un poids de $50 \mathrm{~kg}$; d'où la nécessité de lester fortement: le poids supplémentaire a été fourni par les armatures de manutention en métal plein et par les pieds lestés amovibles de $30 \mathrm{~kg}$ chacun. L'ensemble pèse alors environ $100 \mathrm{~kg}$. Notons que ce problème limite toute réalisation utilisant des ensembles de fort volume et qu'au contraire les améliorations viendront d'une miniaturisation plus poussée.

Les premiers enregistrements obtenus feront l'object d'un travail ultérieur, mais il semble possible dans des conditions de mer calme ou peu agitée de commencer une prospection de routine dans le domaine littoral.

Une question plus générale reste le traitement des données obtenues par les méthodes statistiques. Si nous voulons conserver notre désir primitif, à savoir le point de vue biologique, il faut condenser l'énorme masse de renseignements qui risque d'être rapidement accumulée. La méthode analogique est ici un peu déficiente à cet égard et devrait normalement laisser la place à des convertisseurs analogiques numériques soit à la lecture des bandes d'enregistreurs, soit directement dans la station de mesure.

\section{RÉSUMÊ}

1. Notre objectif a été de réaliser une station relativement simple et peu onéreuse à l'usage des biologistes marins.

2. Cette unité de mesure destinée à travailler à des profondeurs de 0 à $10 \mathrm{~m}$ s'avère susceptible de réaliser des relevés systématiques tout en pouvant recevoir un programme de mesures de paramètres adaptable à chaque problème et à chaque chercheur.

\section{LITTÉRATURE CITÉE}

Benard, F., 1967. Essai de mesure des facteurs écologiques dans la zone des marées. Bull. Inst. océanogr. Monaco (souspresse).

Lacroix, P. \& Laubier, L., 1964. Description, étalonnages et essais préliminaires d'un nouveau courantomètre enregistreur. Cah. océanogr. 16 (9), 726-753. 